\section{Die Aufklärung des Patienten bei Bluttransfusionen}

\begin{abstract}
Zusammenfassung: Wenn Bluttransfusionen während oder nach einer Operation notwendig werden könnten, muß sowohl der verantwortliche Anästhesist als auch der verantwortliche Operateur - entweder jeder für sich oder nach Absprache einer von beiden - den Patienten darüber und besonders über mögliche Folgewirkungen der Transfusion aufklären. Die Durchführung der Aufklärung kann delegiert werden (z. B. Stationsarzt). Kommt als Alternative zur Fremdblutransfusion eine Eigenblutspende in Frage, muß die Aufklärung diese Möglichkeit mit erfassen. Ein tumorerkrankter Patient ist über die Möglichkeit der Eigenblutspende auch dann aufzuklären, wenn diese Möglichkeit von der Bluttransfusionszentrale abgelehnt wird, mit der die ihn behandelnde Klinik zusammenarbeitet.
\end{abstract}

Obtaining the Patients' Informed Consent Before Performing Blood Transfusions: If blood transfusions become necessary during or after surgery the patient must be informed for the purpose of obtaining his informed consent. Such information must be given either by the responsible anaesthesiologist or surgeon - either separately or by agreement by one of them - with particular reference to possible sequels to the transfusion or adverse reactions. The task of informing the patient may also be delegated e.g. to the ward physician. If autohaemotransfusion is considered instead of immediate or indirect transfusion, information must include this possibility. A tumour patient must be informed of the possibility of an autologous transfusion even if this is refused by the blood bank cooperating with the hospital.

Die Bluttransfusion galt als bisher medizinisch unproblematische Behandlungsmethode, die auch keine besonderen Rechtsprobleme enthält. Spätestens seit dem Blut-AIDS-Skandal von 1994 wurde schlagartig deutlich, daß diese Ansicht nicht länger haltbar ist. Die sich aus dem Skandal ergebene Diskussion führte jetzt zu einer gesetzlichen Regelung: Auf Empfehlung des Fachausschusses ${ }^{1}$ beschloß der Bundestag am 7.5.1998 die Annahme des Transfusionsgesetzes der Bundesregierung. ${ }^{2}$ Das Gesetz zur Regelung des Transfusionswesens (Transfusionsgesetz - TFG) ${ }^{3}$ trat am 7. 7. 1998 in Kraft, §39 TFG. Ziel des TFG ist, das Risiko der Übertragung von Infektionskrankheiten durch Blutprodukte zu vermindern und der Selbstversorgung mit Blut und Plasma in Deutschland mehr Geltung zu verschaffen, $\S 1$ TFG.

Akt. Urol. 29 (1998) $273-283$

(c) Georg Thieme Verlag Stuttgart · New York
RA Dr. Klaus Wasserburg

unter Mitwirkung von Annette Clitsch
Unabhängig davon hatte sich vor Inkrafttreten des TFG bei den Urologen und Gynäkologen eine Diskussion zur Blutspende entwickelt, die vor allem Aufklärungsprobleme betrifft, insbesondere Fragen zur Aufklärung des Patienten bei der Eigenbluttransfusion. Die Fragen der Urologen und Gynäkologen wurden anläßlich der Jahrestagung der Deutschen Gesellschaft für Urologie (1997) und der Deutschen Gesellschaft für Gynäkologie und Geburtshilfe (1997) erörtert: Die Tagungsteilnehmer diskutierten über die „Eigenblutspende": Grundsätzlich gilt in der Medizin die Eigenblutspende als sicherste und risikoärmste Form der Blutübertragung, weil sie vor allem die Gefahr der Infektion mit Krankheiten wie Hepatitis und AIDS unmöglich macht ${ }^{4}$. Unsicherheit bestand in der Diskussion aber insbesondere über Inhalt und Umfang der ärztlichen Aufklärungspflicht: Zwar war man sich darüber einig, daß z. B. bei einem urologischen Fall, der einen Tumorerkrankten betrifft, der Operateur den Patienten über die Möglichkeit der intra- oder postoperativen Transfusion von Blut oder Blutprodukten im Rahmen der Aufklärung über den operativen Eingriff selbst aufklären muß und daß der Narkosearzt im Rahmen seines Aufklärungsgespräches den Patienten ebenfalls über die Transfusion und begleitende Risiken informiert. Die Fachgesellschaften waren sich aber nicht darüber einig, ob bei Patienten mit einer Tumorerkrankung Eigenblutspenden kontraindiziert sind oder nicht. Die Vorstellung war, daß bei manchen Tumorerkrankungen Tumorzellen im peripheren Blut zirkulieren, die dem Patienten bei einer notwendig werdenden Eigenbluttransfusion wiedergegeben werden würden. Das Verfahren ist umstritten: Zahlreiche Transfusionszentralen in Deutschland führen auch bei Tumorpatienten Eigenblutentnahmen durch, während andere Transfusionszentralen das ablehnen. Vor diesem Hintergrund wurde von den Teilnehmern der Jahrestagung insbesondere die ärztliche Aufklärungspflicht kontrovers beurteilt, die Ärzte wollten wissen, wie sie sich verhalten sollen, ob der Patient über die Möglichkeit der Eigenblutspende unterrichtet werden muß oder nicht.

Außerhalb der Jahrestagungen wurden Urologen mit einem Fall konfrontiert, der die Diskussion um die Aufklärungspflicht im Rahmen der Eigenblutspende abermals anfachte: Der Patient wurde radikal prostatektomiert und erhielt intraoperativ vier Erythrozytenkonzentrate aufgrund von profusen Blutungen. Nachdem sich eine Hepatitis entwickelt hatte, machte er Schadensersatzansprüche zunächst gegen die Transfusionszentrale mit der Behauptung geltend, die Konserven seien

\footnotetext{
BT-Drucks. 13/10643

BT-Drucks. 13/9594

BGBI. 1998 I Nr. 42, S. 1752
}

${ }^{4}$ BGH NJW 1994, $743 \mathrm{ff}$. 
nicht sorgfältig genug kontrolliert worden. Wäre dies geschehen, wäre eine Hepatitis C-Infizierung der Konserven erkannt worden. Nach Widerlegung dieser Behauptung durch die Transfusionszentrale (das Risiko der Hepatitis C-Infektion durch Blutkonserven liegt bei $1: 30000$ ), stützte sich der Patient, wie in Fällen behaupteter Kunstfehler üblich, auf angeblich ungenügende Aufklärung, indem er vortrug, er sei nicht über die Möglichkeit der Eigenblutspende aufgeklärt worden. Hätte er diese Möglichkeit gehabt, hätte er niemals in eine Fremdbluttransfusion eingewilligt. Die diesem Patienten zur Aufklärung gegebenen Merkblätter für die Patientenaufklärung enthalten keine Hinweise auf die Eigenblutspende, der Patient wurde nicht von dem behandelnden Arzt über die Möglichkeit der Eigenblutspende aufgeklärt. Trotz des Alters des Patienten ( 70 Jahre) gab es keine Kontraindikation individueller Art zur Eigenblutspende. Andere Kliniken hätten in diesen Fällen zwei bis drei Konserven Eigenblut für die Operation bereitgestellt. Da die Eigenblutspende jedoch im betroffenen Klinikum bei Tumorpatienten wegen der ablehnenden Einstellung der Transfusionszentrale nicht möglich war, wurde der Patient nicht entsprechend informiert.

Bei der Beantwortung dieser Fragen ist danach zu differenzieren, ob es um die Aufklärung des Spenders geht oder um die des Patienten, dem das Blut infundiert wird. Beim Patienten ist zu differenzieren zwischen der Aufklärung über die Eigenblutspende und der Aufklärung über die Folgen der Transfusion.

\section{Die Aufklärung des Blutspenders}

In $\S 6$ TFG werden Aufklärung und Einwilligung für den Fall der Spendeentnahme wie folgt geregelt:

(1) Eine Spendeentnahme darf nur durchgeführt werden, wenn die spendende Person vorher in einer für sie verständlichen Form über Wesen, Bedeutung und Durchführung der Spendeentnahme und der Untersuchungen sachkundig aufgeklärt worden ist und in die Spendeentnahme und die Untersuchungen eingewilligt hat. Aufklärung und Einwilligung sind von der spendenden Person schriftlich zu bestätigen. Sie muß mit der Einwilligung gleichzeitig erklären, daß die Spende verwendbar ist, sofern sie nicht vom vertraulichen Selbstausschluß Gebrauch macht.

\section{(2) $\ldots “$.}

In der Begründung zu $§ 6$ TFG wird dazu ausgeführt:

„... Die Vorschrift verlangt vor der Spendeentnahme eine umfassende Aufklärung der spendenden Person über das Entnahmeverfahren, die auch mögliche Risiken umfaßt, die notwendigen Untersuchungen und den Umgang mit spenderbezogenen Daten. Die spendende Person erhält dadurch Kenntnis über Wesen, Bedeutung und Durchführung der Spendeentnahme und die Untersuchungen und kann frei entscheiden, ob sie spenden möchte oder nicht. Die Aufklärung muß in einer für die spendende Person verständlichen Form erfolgen, also auf die Person abgestellt sein. Sachkundig ist die Aufklärung. wenn sie von einer ärztlichen Person oder einer entsprechend geschulten Person durchgeführt wird.
Die spendende Person muß die Aufklärung schriftlich bestätigen, damit ihr das Geschehen voll bewußt wird. Dies kann zusammen mit der Einwilligungserklärung geschehen. Die Spendebereitschaft muß bei jeder Spendeentnahme bestätigt werden. Die jedesmalige Einwilligung der spendenden Person ergibt sich zwingend aus dem Gebot der Freiwilligkeit der Spendeentnahme, die ein seit Jahren international anerkannter Grundsatz ist. Die $\S \S 1626 \mathrm{ff}$. und $1901 \mathrm{ff}$. BGB bleiben unberührt, sofern die Spendeentnahme bei Minderjährigen oder betreuten Personen erfolgt.

Mit der Einwilligung wird gleichzeitig die Verwendbarkeit der Spende erklärt. Dies folgt aus dem Eigentumsrecht nach Artikel 14 Abs. 1 Satz 1 des Grundgesetzes. Die Erklärung zur Verwendbarkeit der Spende gilt nicht, wenn die spendende Person vom vertraulichen Selbstausschluß Gebrauch macht. Der vertrauliche Selbstausschluß wird zugelassen, um Personen, deren Spende nicht verwendet werden darf, die aber in einer Gruppe zur Entnahme kommen, vor Diskriminierung zu schützen."

Für die Aufklärung des Blutspenders ergeben sich nach $\S 6$ TFG keine besonderen Probleme. Sie steht in Einklang mit der bisherigen Rechtsprechung.

\section{Die Aufklärung des Patienten über die Möglichkeit der Eigenblutspende und die Folgen einer Bluttransfusion}

Bei der Aufklärung des Patienten sind zwei Aufklärungsfelder zu beachten: zum einen die Aufklärung über die Eigenblutspende, zum anderen die Aufklärung über die Folgen einer Bluttransfusion.

\section{Die Aufklärung des Patienten über die Möglichkeit der Eigenblutspende}

Bei der Eigenblutspende ist zu trennen zwischen der Aufklärung über die Möglichkeit der Eigenblutspende im Einzelfall überhaupt und dem Sonderfall der Möglichkeit der Eigenblutspende beim tumorerkrankten Patienten.

\section{Die grundsätzliche Aufklärung über die Eigenblutspende}

Das TFG hat die Frage, ob und inwieweit Patienten über die Möglichkeit einer Eigenbluttransfusion aufzuklären sind, geregelt. Die Regelung wurde bei den Anforderungen an die Durchführung der Anwendung der Blutprodukte in $\S 13$ Abs. 1 TFG aufgenommen:

(1) Blutprodukte sind nach dem Stand der medizinischen Wissenschaft und Technik anzuwenden. Es müssen die Anforderungen an die Identitätssicherung, die vorbereitenden Untersuchungen, einschließlich der vorgesehenen Testung auf Infektionsmarker und die Rückstellproben, die Technik der Anwendung sowie die Aufklärung und Einwilligung beachtet werden. Ärztliche Personen, die im Zusammenhang mit der Anwendung von Blutprodukten Laboruntersuchungen durchfüren oder anfordern, müssen für diese Tätigkeiten besonders sachkundig sein. Die Anwendung von Eigenblut richtet sich auch nach den Besonderheiten dieser Blutprodukte. Die zu behan-

${ }^{5}$ BT-Drucks, 13/9594, S. 18 
delnden Personen sind, soweit es nach dem Stand der medizinischen Wissenschaft vorgesehen ist, über die Möglichkeit der Anwendung von Eigenblut aufzuklären.

\section{(2) $\ldots$}

Diese Regelung verlangt also, daß die zu behandeinden Personen, soweit es „nach dem Stand der medizinischen Wissenschaft" vorgesehen ist, über die Eigenblutspende aufzuklären sind. Damit ist die Ausgangsfrage der Ärzte nach Erforderlichkeit der Aufklärung über die Eigenblutspende im Grundsatz beantwortet.

Die Notwendigkeit der Aufklärung hängt jedoch nach §13 TFG von dem "Stand der medizinischen Wissenschaft" ab.

Zum Begriff "Stand der medizinischen Wissenschaft" bestimmt $§ 18$ Abs. 1 TFG folgendes:

„(1) Die Bundesärztekammer stellt im Einvernehmen mit der zuständigen Bundesoberbehörde und nach Anhörung von Sachverständigen unter Berücksichtigung der Empfehlungen der Europäischen Union, des Europarates und der Weltgesundheitsorganisation zu Blut- und Blutbestandteilen in Richtlinien den allgemein anerkannten Stand der medizinischen Wissenschaft und Technik insbesondere für

1....

$2 \ldots$

$3 \ldots$

$4 \ldots$

fest $(\ldots)^{\prime \prime}$.

Die Richtlinien der Bundesärztekammer (BÄK) wurden bisher noch nicht entsprechend dem TFG überarbeitet, d.h., die Richtlinien befinden sich auf dem Stand des Jahres 1996.

Es läßt sich damit nur feststellen, daß grundsätzlich über die Möglichkeit der Eigenblutspende aufzuklären ist, soweit das was der Arzt im Einzelfall feststellen muß - vom Stand der medizinischen Wissenschaft verlangt wird.

Bei der Diskussion des Gesetzentwurfes sah man in diesem etwas verschwommenen Begriff keine größeren Probleme, lediglich die - für die Aufklärung nicht relevante - Verbindung des Begriffs mit dem Stand der Technik wurde kritisiert ${ }^{6}$.

In der Begründung des Gesetzes wird zu §13 TFG u.a. ausgeführt:

„Die Anwendung von Eigenblut richtet sich nach deren Besonderheiten. So kann es z. B. im Einzelfall begründet sein, infektiöses Eigenblut zu infundieren. Im übrigen werden die behandelnden ärztlichen Personen verpflichtet, die zu behandelnden Personen in den nach dem Stand der medizinischen Wissenschaft vorgesehenen Fällen auf die Möglichkeit der Eigenblutspende aufmerksam zu machen. Dies entspricht höchstrichterlicher Rechtsprechung (vgl. Entscheidung des Bundesgerichtshofes vom 17. Dezember 1992 - Az.: VI/ZR 4091)."

\footnotetext{
${ }^{6} \mathrm{vgl}$. Knoche BT-Drucks. 13/21652

${ }^{7}$ BT-Drucks. 13/9594. S. 22
}

Aus dieser Begründung ergibt sich zweierlei: Nach der Begründung kann infektiöses Eigenblut im Einzelfall infundiert werden. Wenn aber infektiöses Eigenblut infundiert werden darf, kann wohl auch davon ausgegangen werden, daß Eigenblut mit Krebszellen infundiert werden darf, wenn das „im Einzelfall begründet ist"; ob das der Fall ist, ist eine medizinische Frage.

Der Begriff "Stand der medizinischen Wissenschaft“ wird nicht in der Begründung zu § 13 TFG definiert. In der in der Begründen zu § 13 TFG genannten Entscheidung des BGH wird hierzu als Leitsatz ausgeführt:

„Patienten sind immer dann über das Risiko einer Infektion mit Hepatitis und AIDS bei der Transfusion von Fremdblut aufzuklären, wenn es für den Arzt ernsthaft in Betracht kommt, daß bei ihnen intra- oder postoperativ eine Bluttransfusion erforderlich werden kann. Darüber hinaus sind solche Patienten auf den Weg der Eigenblutspende als Alternative zur Transfusion von fremdem Spenderblut hinzuweisen, soweit für sie diese Möglichkeit besteht"8.

Diese Entscheidung liegt auf der Linie der bisherigen Rechtsprechung, nämlich dem Gebot, auch über seltene, schwere, spezifische und für den Laien überraschende Risiken aufzuklären. Der Begriff "Stand der medizinischen Wissenschaft“ wird aber auch hier nicht eingegrenzt.

Zum Begriff „Stand der medizinischen Wissenschaft" verweist die Begründung zu § 12 TFG auf die Begründung zu $\S 5 \mathrm{TFG}^{9}$. Die Begründung zu $§ 5$ TFG führt u.a. aus:

„Der Begriff ,Stand der medizinischen Wissenschaft und Technik' wird nahezu durchgängig im Gesetzentwurf verwendet, um herauszustellen, daß der Gesetzentwurf in erster Linie und überwiegend medizinische oder die Ärzteschaft betreffende Sachverhalte regelt. Sie werden ausgefüllt und konkretisiert durch die Erkenntnisse der medizinischen Wissenschaft und Technik. Diese ergeben sich nach der Konzeption des Gesetzentwurfes als allgemein anerkannter Standard in der Regel aus den Richtlinien der Bundesärztekammer (vgl. §12). Sie können sich auch aus Empfehlungen des Arbeitskreises Blut des Bundesministeriums für Gesundheit ergeben, bis Richtlinien der Bundesärztekammer erstellt oder überarbeitet worden sind. Sie konkretisieren sich im Einzelfall verbindlich durch Auflagen der zuständigen Bundesoberbehörde nach dem Arzneimittelgesetz." 10

Aus alledem folgt zusammenfassend, daß der Patient regelmäBig über die Möglichkeit der Eigenblutspende aufzuklären ist, es sei denn, sie ist medizinisch ausgeschlossen. Der Begriff in $\S 13$ Abs. 1 TFG: „Stand der medizinischen Wissenschaft" trägt jedenfalls nicht zur Klarheit bei ${ }^{11}$.

Möglicherweise könnte das Konzept der „evidence-based-medicine" helfen. Das Konzept soll es dem praktizierenden Arzt erleichtern, seine Patienten nach den neuesten, wissenschaftlich

\footnotetext{
${ }^{8}$ BGH MedR 1992, S. 159

${ }^{9}$ BT-Drucks. 13/9594, S. 21, 17

${ }^{10}$ BT-Drucks. 13/9594, S. 17

11 vgl. zu alledem: Rohde. NJW 1988, S. 2285, m.w.N
} 
fundierten Erkenntnissen zu behandeln. Ärzte sind oft bei der Beschaffung der erforderlichen Materialien überfordert ${ }^{12}$.

\section{Die Aufklärung des tumorerkrankten Patienten}

Die Diskussion der Ärzte betraf auch das besondere Problem der Aufklärung des tumorerkrankten Patienten. Die Frage, ob der Arzt den Patienten auch über Behandlungsalternativen, wie sie die Eigenblutspende gegenüber der Fremdbluttransfusion darstellt, unterrichten muß, gehört zur Alternativaufklärung. Die Information über Behandlungsalternativen ist ein Hauptanwendungsfall und wird als wichtiger Bewährungsfall des Selbstbestimmungsrechts des Patienten bezeichnet. ${ }^{13}$ Hierzu hat der Bundesgerichtshof festgestellt:

„Stehen für eine medizinisch sinnvolle und indizierte Therapie mehrere Behandlungsmethoden zur Verfügung, die zu jeweils unterschiedlichen Belastungen des Patienten führen oder unterschiedliche Risiken und Erfolgschancen bieten, muß der Patient - selbstverständlich nach sachverständiger und verständnisvoller Beratung des Arztes - selbst prüfen können, was er an Belastungen und Gefahren im Hinblick auf möglicherweise unterschiedliche Erfolgschancen der verschiedenen Behandlungsmethoden auf sich nehmen will." ${ }^{4}$

Auf der Basis dieser Feststellungen gilt für die Eigenblutspende grundsätzlich: Die Medizin sieht die Eigenblutspende als die sicherste und risikoärmste Form der Blutübertragung an, weil sie vor allem die Gefahr der Übertragung von Krankheiten, wie Hepatitis und AIDS, unmöglich macht. Deshalb muß der Patient, soweit diese Möglichkeit für ihn besteht, und wie es $\S 13$ TFG verlangt, auch nach der bisherigen Rechtsprechung auf die Möglichkeit der Eigenblutspende als Alternative zur Transfusion fremden Spenderbluts hingewiesen werden. ${ }^{15} \mathrm{Er}$ ist nach den Grundsätzen zur Aufklärung über Behandlungsalternativen auch über die Vorzüge und Nachteile der beiden Transfusionsmethoden zu informieren, es muß mit ihm abgestimmt werden, ob die Transfusion mit eigenem oder fremdem Blut durchgeführt werden soll. ${ }^{16}$

Wie eingangs erwähnt, sind sich die Mediziner nicht darüber einig, ob bei Patienten mit einer Tumorerkrankung Eigenblutspenden, weil sie eine Streuung von Krebszellen bewirken könnten, kontraindiziert sind oder nicht - einige Transfusionszentren lehnen die Eigenblutentnahme bei Tumorpatienten $a b$, andere führen sie durch. Es hängt also von der jeweiligen medizinischen Einschätzung der Frage durch die einzelnen Zentralen ab, ob die krebserkrankten Patienten in ihrem Einzugsbereich Eigenblutransfusionen erhalten können oder nicht. Die Eigenbluttransfusion stellt sich als eine medizinisch umstrittene Behandlungsmethode dar. Wenn sie eine medizinisch vertretbare Behandlungsalternative ist, die dem Patienten eine echte Wahlmöglichkeit eröffnet, muß er über die Möglichkeit unterrichtet werden. Da aber bei der Eigenbluttransfusion die Gefahr der Streuung von Krebszellen im Ge-

\footnotetext{
12 vgl. dazu: Perleth, Matthias, Anthes, Gerd (Hrsg.). „Evidenzbasierte Medizin. Wissenschaft im Praxisalltag." München 1998

${ }^{13}$ Damm, NJW 1989, 737 ff. (741)

${ }^{14}$ BGHZ 102, 17 ff. (22)

${ }^{15}$ BGH. NJW 1992, 743 ff. (744)

${ }^{16}$ BGH, NJW 1992, 743 ff. (744)
}

gensatz zu den Infektionsgefahren bei der homologen Bluttransfusion besteht, sind unterschiedliche Risiken vorhanden, die dem Patienten eine Wahlmöglichkeit geben. Der Patient muß selbst prüfen können, was er an Belastungen und Gefahren im Hinblick auf möglicherweise unterschiedliche Erfolgschancen der verschiedenen Behandlungsmethoden auf sich nehmen will. ${ }^{17}$ Zusätzlich muß er auch auf die Tatsache hingewiesen werden, daß die alternative Therapie in der medizinischen Wissenschaft umstritten ist. Möchte der Arzt nach einer ernsthaft umstrittenen Methode behandeln, würde er etwa bei dem Tumorpatienten die Eigenblutspende durchführen wollen, so muß er den Patienten im Aufklärungsgespräch darüber informieren, daß gewichtige Stimmen in der Wissenschaft vor dieser Vorgehensweise warnen. ${ }^{18}$ Der Grund dieser Informationspflicht ergibt sich aus den Grundsätzen, die zur Sicherung der Selbstbestimmung des Patienten unabdingbar sind. ${ }^{19}$

Unterrichtet der Arzt im Aufklärungsgespräch über eine Behandlungsalternative zu der von ihm vorgeschlagenen Therapie, so muß er auf ernstzunehmende Ansichten, die gegen die alternative Methode sprechen, selbstverständlich ebenso hinweisen. Zudem verlangt das Gebot der Alternativaufklärung, daß der Arzt mit dem Patienten die Vorzüge und Nachteile der verschiedenen Möglichkeiten besprechen soll. ${ }^{20}$ Ernstzunehmende Warnungen in der Wissenschaft gegen die Eigenblutspende wegen einer Gefahr möglicher Kontraindikation zählen zu diesen Nachteilen. Auch für andere umstrittene Behandlungsalternativen, etwa für das Hämodilutionsverfahren, gilt nichts anderes als das, was zur Eigenblutspende ausgeführt wurde. ${ }^{21}$

\section{Die Aufklärung des Patienten über mögliche Folgen einer Bluttransfusion}

Das TFG enthält zur Aufklärung bei Bluttransfusionen lediglich die oben dargestellten Regelungen. Die Aufklärung des Patienten über die Folgen einer möglicherweise oder sicher erforderlich werdenden Bluttransfusion richtet sich hingegen mangels gesetzlicher Regelung nach wie vor nach den allgemeinen Grundsätzen über die ärztliche Aufklärungspflicht. Dabei ist allerdings zu überlegen, ob einzelne Regelungen des TFG eine Änderung der bisherigen Praxis gebieten.

Da die Frage der Aufklärung ständig in der ärztlichen Praxis diskutiert wird und die von der Rechtsprechung geforderte Aufklärung schon seit einem Jahrhundert zwischen Ärzten und Juristen Konflikte verursacht, soll nachfolgend auf ihre Entwicklung eingegangen werden, sodann auf ihre dogmatische Begründung, auf ihre Formen und ihre Systematisierung.

\footnotetext{
17 vgl. BGHZ 102, 17 ff. (22); Scholz, MDR 1996, 649 ff.

${ }^{18} \mathrm{BGH}$, MedR 1996, $271 \mathrm{ff}$ (271), BGH, NJW 1982, 578 ff; BGH, NJW 1978,587 ff. (588)

${ }^{19} \mathrm{BGH}$, NJW 1978, 587 ff. (588)

${ }^{20}$ BGH. NJW 1992. 643 ff. (644)

${ }^{21}$ kritisch dazu: Weißauer, Opderbecke, MedR 1992, $307 \mathrm{ff}$.
} 
Die Entwicklung der Aufklärungspflicht in der Rechtsprechung seit 1894

Die erste für die Aufklärungspflicht grundlegende Entscheidung des Reichsgerichts liegt schon über 100 Jahre zurück; es ging in dieser Strafsache um einen Fall eigenmächtiger Heilbehandlung: In der Entscheidung des Reichsgerichts vom 31. 5. 1894 wurde der Arzt wegen Körperverletzung verurteilt, weil er einem 7 Jahre alten Mädchen, das an einer tuberkulösen Vereiterung der Fußwurzelknochen litt, den Fuß amputiert hatte, obwohl ihm der Vater als gesetzlicher Vertreter erklärt hatte, daß er die beabsichtigte Operation nicht erlaube. ${ }^{22}$ Der Vorwurf betraf also die Durchführung der Operation trotz fehlender Einwilligung des Sorgeberechtigten. Die Aufklärungspflicht als Voraussetzung für die Erteilung einer wirksamen Einwilligung wurde in dieser Entscheidung noch nicht angesprochen, ebensowenig in den weiteren Entscheidungen aus dieser Zeit, die sich mit Fällen eigenmächtiger Heilbehandlung zu befassen hatten, obwohl die Aufklärung Voraussetzung für eine wirksame Einwilligung des Patienten ist.

In einer weiteren zivilrechtlichen Entscheidung des RG vom 1. 3. 1912 steht das Gericht der ärztlichen Aufklärung noch sehr kritisch gegenüber; ${ }^{23}$ dort wird ausgeführt:

„Die Annahme einer derartigen Verpflichtung läßt sich weder aus der Übung der pflichtgetreuen und sorgfältigen Vertreter des ärztlichen Berufes, noch aus inneren Gründen herleiten. Eine umfassende Belehrung des Kranken über alle möglichen Folgen der Operation würde nicht selten sogar falsch sein, sei es, daß der Kranke dadurch abgeschreckt wird, sich der Operation zu unterwerfen, obwohl sie trotz der damit verbundenen Gefahren geboten oder doch zweckmäßig ist, sei es, daß der Kranke durch die Vorstellung der mit der Operation verbundenen Gefahren in Angst und Erregung versetzt und so der günstige Verlauf der Operation und der Heilung gefährdet wird." ${ }^{24}$

Das Gericht stellt hier den Schonungsgrundsatz über die Aufklärungspflicht und gewährt damit dem Arzt einen rechtlich weitreichenden Freiraum, der in der Folgezeit immer mehr zugunsten der Entscheidungsfreiheit des Patienten eingeengt wurde. ${ }^{25}$

In den 30er Jahren hatte das Reichsgericht Fälle von Schadensersatzansprüchen wegen Verletzung der Aufklärungspflicht zu entscheiden. Das Gericht bejahte das Bestehen der Ansprüche, jedoch hielt sich die Begründung noch sehr zurück, weil man durch die Aufklärung den Heilerfolg nicht gefährden wollte; das Gericht räumte dem Arzt einen gewissen Ermessensspielraum ein, einen gleichsam „rechtsfreien“ Raum, in dem der Arzt Art und Umfang der Aufklärung bestimmen konnte. ${ }^{26}$

In der Nachkriegszeit verstärkte die Rechtsprechung zunehmend die Aufklärungspflicht. Sie wird mit dem Selbstbestim-

\footnotetext{
${ }^{22}$ RGSt 25,375

${ }^{23}$ RGZ 78, 432

${ }^{24}$ RGZ 78, 432, 433f. - vgl. dort auch zu Fragen der Beweislast bei Eintritt schädlicher Folgen durch die Operation

${ }^{25} \mathrm{vgl}$. dazu: Roßner, Begrenzung der Aufklärungspflicht des Arztes bei Kollision mit anderen ärztlichen Pflichten, Frankfurt 1998.

${ }^{26} \mathrm{vgl}$. dazu besonders: Tempel NJW 1980, 609 ff.; RG JW 1932, 3328 und 3369, RG JW 1937, 3087
}

mungsrecht des Patienten begründet, mit dem in Art. 2 II GG gewährleisteten Persönlichkeitsrecht. In einer unter der Bezeichnung Myom-Urteil bekanntgewordenen Entscheidung des $\mathrm{BCH}^{27}$ findet sich die wesentliche Begründung dafür, daß der Arzt nur im Rahmen des Einverständnisses des Patienten handeln darf.

„Sollte sich ergeben, daß der Angeklagte sich dieser Möglichkeit hätte bewußt sein müssen, so wäre weiter zu prüfen, ob er dann nicht verpflichtet war, seine Patienten auf diese Möglichkeit hinzuweisen und sich ihres Einverständnisses zu versichern. Dieser Pflicht wäre er nicht deshalb enthoben gewesen, weil ег eine doppelfaustgroße Geschwulst festgestellt hatte und die Nebenklägerin dies vor der Operation wußte. Diese Tatsache rechtfertigte nicht ohne weiteres den Schluß des Angeklagten, die Patientin werde schon mit der Entfernung ihrer Gebärmutter einverstanden sein, wenn allein dadurch die Geschwulst beseitigt werden konnte. Denn es ist möglich, daß ein Kranker eine selbst gefährliche Geschwulst an einem Organ seines Körpers eher weiterbestehen zu lassen bereit ist, als für die Beseitigung der Geschwulst den Verlust eines ganzen Organs in Kauf zu nehmen. Mag diese Entscheidung für ihn selbst lebensbedrohlich und deshalb jedenfalls dann unverständlich sein, wenn er auch ohne das Organ weiterleben könnte, so muß sie doch von jedem, auch einem Arzt, in Betracht gezogen und beachtet werden. Das in Art. 2 II 1 GG gewährleistete Recht auf körperliche Unversehrtheit fordert Berücksichtigung auch bei einem Menschen, der es ablehnt, seine körperliche Unversehrtheit selbst dann preiszugeben, wenn er dadurch von einem lebensgefährlichen Leiden befreit wird. Niemand darf sich zum Richter in der Frage aufwerfen, unter welchen Umständen ein anderer vernünftigerweise bereit sein sollte, seine körperliche Unversehrtheit zu opfern, um dadurch wieder gesund zu werden. Diese Richtlinie ist auch für den Arzt verbindlich. Zwar ist es sein vornehmstes Recht und seine wesentlichste Pflicht, den kranken Menschen nach Möglichkeit von seinem Leiden zu heilen. Dieses Recht und diese Pflicht finden aber in dem grundsätzlichen freien Selbstbestimmungsrecht des Menschen über seinen Körper ihre Grenze. Es wäre ein rechtswidriger Eingriff in die Freiheit und Würde der menschlichen Persönlichkeit, wenn ein Arzt - und sei es auch aus medizinisch berechtigten Gründen - eigenmächtig und selbstherrlich eine folgenschwere Operation bei einem Kranken, dessen Meinung rechtzeitig eingeholt werden kann, ohne dessen vorherige Billigung vornähme. Denn ein selbst lebensgefährlich Kranker kann triftige und sowoh! menschlich wie sittlich achtenswerte Gründe haben, eine Operation abzulehnen, auch wenn er durch sie und nur durch sie von seinem Leiden befreit werden könnte." 28

Damit wurde der frühere ärztliche „Freiraum“ des Reichsgerichts aufgegeben, der Arzt ist seither unter Androhung einer Bestrafung wegen Körperverletzung verpflichtet, die Autonomie des Patienten umfassend zu respektieren, ihn aufzuklären

\footnotetext{
${ }^{27}$ BGH St $11,111,113$

${ }^{28}$ BHG St 11, 113 f. unter Hinweis auf Eb. Schmidt, in: Ponsold, Lehrbuch 1957, S. 37
} 
und nur im Rahmen seiner Einwilligung zu handeln. Maß und Umfang der geschuldeten Aufklärung werden bestimmt von Häufigkeit und Schwere der auftretenden Komplikationen; die Aufklärung ist abhängig von der Gefährlichkeit der ärztlichen Behandiung. Der Patient muß über die "typischen“ Behandlungsgefahren aufgeklärt werden. nicht aber über äuBerst seltene Schadensfolgen. Der Aufklärungsumfang wird zur allein von den Gerichten zu bestimmenden Rechtsfrage, der Arzt hat jeden Ermessensspielraum verloren. Eine „restlose" Aufklärung ist nur bei ernsthafter Gefahr für Leib oder Leben des Patienten nicht erforderlich. ${ }^{29}$ Die potentielle gesundheitliche Gefährdung des Patienten durch die Aufklärung hat kaum noch eine Bedeutung.

Ursache dieser Entwicklung sind wohl u.a. die großen Fortschritte in der medizinischen Wissenschaft, gerade vor und seit der Jahrhundertwende, die zur Folge hatten und haben, daß der Patient heute viel eher als früher ohne Aufklärung oft nicht mehr weiß, was überhaupt mit ihm geschieht. Hinzu kommt die Beeinflussung des Patienten durch die Medien. Viele Patienten sind medizinisch gesehen "halbgebildet", sie schätzen ihre gesundheitliche Lage oft nicht richtig ein. Das Verhältnis zwischen Arzt und Patient hat sich deshalb und aus vielen anderen Gründen erheblich gewandelt: An die Stelle des Vertrauens des Kranken auf die Autoritat des Arztes und dessen Können ist eine Verrechtlichung des Arzt-Patientenverhältnisses getreten, das zwar auch von Vertrauen geprägt sein sollte, jedoch ist das oft nicht der Fall: Sobald das Vertrauen fehlt, kommt es zum Konflikt.

Vor dem Hintergrund dieser sehr komplex verlaufenden und hier nur in wenigen Worten skizzierten Entwicklung muß die Aufklärungspflicht gesehen werden.

\section{Begründung der Aufklärungspflicht}

Die - in der Literatur häufig und oft zu Recht - kritisierte Rechtsprechung bewertet jede ärztliche Behandlung als Körperverletzung, die nur durch eine wirksam erteilte Einwilligung des Patienten gerechtfertigt wird - dafür wiederum ist eine ordnungsgemäße Aufklärung Voraussetzung.

Nach der Rechtsprechung ist jede mit einer Einwirkung auf die körperliche Integrität des Patienten verbundene Heilbehandlung - und zwar auch die medizinisch indizierte und lege artis durchgeführte Behandlung - tatbestandsmäßig eine Körperverletzung, $\S \S 823$ BGB, 223 StGB, die der besonderen Rechtfertigung bedarf; die Rechtfertigung liegt in der Einwilligung des Patienten in die Heilbehandlung. Voraussetzung für die Wirksamkeit der Einwilligung ist eine ordnungsgemäße Aufklärung des Patienten, denn nur derjenige kann wirksam in einen Heileingriff einwilligen, der dessen Wesen, Bedeutung und Tragweite kennt und beurteilen kann und der in der Lage ist. sodann auf der Grundlage der Kenntnis aller maßgeblichen Umstände in die Behandlung einzuwilligen. Bei Kindern, Geisteskranken und Bewußtlosen wird die Einwilligung ersetzt (z. B. durch Einwilligung von Pfleger, Vormund, Erziehungsbe- rechtigte). Die zum Ersatz der Einwilligung Berechtigten müssen dann genau wie der Patient, entweder neben ihm oder an dessen Stelle, aufgeklärt worden sein, es sein denn, es liegt ein Notfall vor mit der Folge, daß die Einhaltung der Einwilligung unmöglich ist.

Eine mangelhafte ärztliche Aufklärung hat eine unwirksame Einwilligung zur Folge, d. h., es liegt eine rechtswidrige Körperverletzung vor. Die Körperverletzung hat zivil- und strafrechtliche Folgen: Der Arzt ist bei einer wegen unzureichender Aufklärung unwirksam erteilten Einwilligung strafrechtlich verantwortlich (Körperverletzung). Zivilrechtlich kann der Patient gegen den Arzt einen Schadensersatzanspruch aus positiver Verletzung des Behandlungsvertrages und außerdem einen deliktischen Schadensersatzanspruch aus $\$ 823$ I BGB gegen den Arzt geltend machen.

An dieser Rechtsprechung wird - neben vielen anderen Aspekten - kritisiert, daß die Behauptung der Aufklärungspflichtverletzung immer erst dann von Patienten vorgetragen werde, $N$ wenn sich der eigentliche Kunstfehlervorwurf nicht beweisen lasse: auch im oben erwähnten urologischen Ausgangsfall war es so, daß sich die Behandlung des Patienten bezüglich einer unzureichenden Kontrolle der Blutkonserve nicht beweisen ließ, infolge dessen wechselte er den Kunstfehlervorwurf ge-ic gen den der Verletzung der Aufklärungspflicht aus. Beide Be-. gründungen können und werden häufig nebeneinander geltend gemacht, um die Chancen eines Erfolges des Schadensersatzanspruches zu erhöhen. Anders als sonst bei Behandlungsfehlern obliegt dem Arzt bei der behaupteten Verletzung der Aufklärungspflicht die Beweislast. Darin liegt für den Patienten ein erheblicher Vorteil.

Für die Bluttransfusion gelten die genannten Grundsätze der Rechtsprechung ebenso wie für jede andere Heilbehandlung: Die Bluttransfusion ist ein Eingriff in die körperliche lntegrität mit nicht unerheblichen Risiken, sie bedarf der Einwilligung $\circlearrowleft$ des Patienten, die nur wirksam ist, wenn er vollständig aufgeklärt wurde. Wenn die Transfusion bei einer konservativen Be-. handlung oder auch prä- oder postoperativ notwendig wird, so: gelten die von der Rechtsprechung zur Selbstbestimmungs- $\frac{\bar{\alpha}}{\alpha}$ aufklärung entwickelten Grundsätze. ${ }^{30}$ Ist die Bluttransfusion vital indiziert und dringend, so kann sich der Umfang der Aufklärung weitgehend und sogar bis auf Null reduzieren. Ist der $\frac{0}{0}$ Patient bewußtlos, so muß der Arzt die Transfusion ohne Aufklärung nach den Grundsätzen der mutmaßlichen Einwilligung durchführen, d.h. der Arzt muß davon ausgehen, daß der Patient eingewilligt hätte, wenn er noch hätte gefragt werden können; hat der Arzt aber gegenteilige Anhaltspunkte (z.B. grundsätzliche Verweigerung der Einwilligung aus religiösen Gründen), dann kann er nicht nach den Regeln der mut- $-\frac{\oplus}{a}$ maßlichen Einwilligung vorgehen. ${ }^{31}$ Die Zeugen Jehovas lehnen beispielsweise die Transfusion aus religiösen Gründen ab. Das muß der Arzt bei solchen Patienten respektieren. Andernfalls liegt eine rechtswidrige Körperverletzung vor. Ob die Rechtsprechung diese Fälle zutreffend löst, ist m.E. zweifelhaft, wenngleich innerhalb der dogmatischen Begründung

${ }^{30}$ Weißauer, MedR 1987, 272

${ }^{31}$ vgl. Weißauer, MedR 1987, 272 ff. vgl. demnächst in MedR 1998 den dort angekündigten Beitrag Benders: Zeugen Jehovas und Bluttransfusion. 
der rechtswidrigen Körperverletzung konsequent: Niemand kann wissen, ob der konkrete bewußtlose Patient, wenn er im Augenblick der Notwendigkeit der Durchführung der Transfusion nochmals hätte gefragt werden können, nicht seine kurz vorher ausdrücklich geäußerte entgegenstehende Meinung geändert und sich entgegen den Regeln seiner Religion doch für die Rettung seines Lebens entschieden hätte.

\section{Die Formen der Aufklärung}

Die Aufklärung läßt sich in einzelne Formen unterteilen, die nachfolgend dargestellt werden, jedoch hat diese Darstellung nur einen skizzenhaften, kursorischen Charakter, im allgemeinen wird von folgenden Formen von Aufklärung gesprochen: ${ }^{32}$

1. Zweck der therapeutischen Aufklärung ist nicht die Verwirklichung des Selbstbestimmungsrechts, sondern die Vermeidung von Gefahren oder Fehlbehandlungen. Ihr Schwergewicht liegt auf Gesundheitshinweisen, Verhaltensmaßregeln, Diätanweisungen, Empfehlungen usw.

2. Die Diagnoseaufklärung betrifft die Unterrichtung des Patienten über den Befund.

3. Die Verlaufsaufklärung informiert den Patienten über die Therapie (d.h. Art, Schwere, Umfang, Durchführung, Schmerzhaftigkeit usw.) des Eingriffs. Teil der Verlaufsaufklärung ist es auch, dem Patienten aufzuzeigen, wie sich sein Gesundheitszustand ohne Durchführung des Eingriffs voraussichtlich entwickeln könnte und welche Folgen und Erfolgschancen die Behandlung (2.B. Operation oder medikamentöse Behandlung) voraussichtlich haben wird.

4. Den medizinischen und juristischen Schwerpunkt bildet die Risikoaufklärung - danach ist der Patient auf sicher eintretende oder auf mögliche Risiken der geplanten ärztlichen Maßnahme und auf sonst mögliche unerwünschte Nebenfolgen sowie auf die Gefahr eines Fehlschlages hinzuweisen. Inwieweit das im Einzelfall unter Beachtung aller konkreten Umstände zu geschehen hat und was alles zum aufklärungsbedürftigen Risiko gehört, ist die praktisch entscheidende und zugleich auch sehr umstrittene Frage. Die Alternativaufklärung kann als Teil der Risikodufklärung angesehen werden, einige sehen sie jedoch als eigenständige Form der Aufklärung an. Nach der Alternativaufklärung ist die Wahl möglicher Behandlungsalternativen solange Sache des Arztes, als es mehrere gleichermaßen erfolgversprechende Behandlungsmöglichkeiten gibt. Deshalb ist über mögliche Behandlungsalternativen nur (aber immerhin) insoweit aufzuklären, als sie mit unterschiedlichen Folgen und Risiken behaftet sind und wenn sie zudem dem Patienten eine echte Wahlmöglichkeit geben können.

Im Rahmen der Risikoaufklärung stellt sich die Frage, ob der Patient auch über evtl. eintretende Nebenfolgen der homologen Blutübertragung aufgeklärt werden muß: Nach der Rechtsprechung muß der Arzt den Patienten über die spezifischen Risiken einer Behandlung aufklären, wenn sie im Falle ihrer

\footnotetext{
32 vgl. z. B. Ulsenheimer, Arztstrafrecht in der Praxis 1988, $50 \mathrm{ff}$ Rdnr. $61 \mathrm{ff.}$ : Eser in: Schönke/Schröder, Strafgesetzbuch, 25. Auflage, 1997 $\S 223$ Rdnr. 41 m.w.N.
}

Verwirklichung seine Lebensführung schwer belasten würden, das Selbstbestimmungsrecht des Patienten verlangt, ihm die mit der gewählten Behandlungsmethode möglicherweise verbundenen Gefahren mitzuteilen, ${ }^{33}$ denn es ist grundsätzlich nicht Sache des Arztes, sondern des Patienten, ob er das mit dem Eingriff verbundene Risiko eingehen will. ${ }^{34}$

Zu den Risiken der Bluttransfusion zählen bisher vor allem immunologisch bedingte Nebenwirkungen sowie bakterielle, virale und parasitäre Infektionen, wobei HIV und Hepatitis nur einzelne aus einer Fülle von Infektionskrankheiten darstellen. ${ }^{35}$ Eine solche Infektion wäre zweifellos geeignet, im Falle ihres Eintritts die Lebensführung schwer zu belasten. Deswegen war bisher die Information des Patienten darüber notwendig. Durch die jetzt im TFG aufgestellten Anforderungen im Rahmen der Blutgewinnung könnte es durchaus möglich sein, daß alsbald bei Einhaltung dieser juristischen Vorgaben die Risiken einer Bluttransfusion für die Patienten weitestgehend minimiert werden, so daß auch die Anforderungen an die Aufklärung des Patienten neu überprüft werden müssen. $§ 5 \mathrm{Abs} .3$ TFG regelt z. B., daß die nach $§ 2$ Abs. 2 Satz 1 der Betriebsordnung für pharmazeutische Unternehmer bestimmte Person dafür zu sorgen hat, daß die spendende Person vor der Freigabe der Spende nach dem Stand der medizinischen Wissenschaft und Technik auf Infektionsmarker, mindestens auf Humanes Immundefekt Virus (HIV)-, Hepatitis B- und Hepatitis C Virus-Infektionsmarker untersucht wird.

Außerdem enthält das TFG Regelungen zu Anforderungen an Spendeeinrichtungen, Auswahl der Spender, Spenderdokumentation. Anforderungen an die Durchführung der Anwendung von Blutprodukten, chargenbezogene Dokumentation der Anwendung von Blutprodukten, Qualitätssicherung der Transfusion, Unterrichtungspflichten der Ärzte bei schwerwiegenden Komplikationen, Verfahren zur Rückverfolgung, Mitteilungspflichten der Behörden und Sachkenntnisvorschriften durch Änderung des Arzneimittelgesetzes.

Es bleibt abzuwarten, ob mit diesen Anforderungen tatsächlich die Risiken der Transfusion für den Empfänger so weitgehend ausgeschlossen werden können, daß die Aufklärung über die mit der Transfusion verbundenen Risiken entfallen würde.

Durch die bisherige Rechtsprechung stellt sich nämlich die Frage nach der Notwendigkeit der Aufklärung besonders dann, wenn die Komplikationsdichte des Infektionsrisikos sehr niedrig ist, wenn z.B. die Wahrscheinlichkeit $1 \%$ oder weniger beträgt, daß überhaupt eine Blutübertragung bei der konkreten Operation erforderlich werden könnte; in diesen Fällen würde die ohnehin sehr niedrige Komplikationsdichte hinsichtlich des Infektionsrisikos noch viel geringer sein. Nach ständiger Rechtsprechung des Bundesgerichtshofes ${ }^{36}$ kommt es aber für die Frage, ob der Patient aufgeklärt werden muß, nicht allein auf die erfahrungsgemäß zu befürchtende Komplikationsdichte an, sondern auf das Gewicht, das mögliche, nicht ganz au-

\footnotetext{
${ }^{33}$ BGH, MedR 1996, $271 \mathrm{ff}$ ( (277)

${ }^{34}$ BGH, MedR 1996, 277 ff. (273)

${ }_{35}$ Weißauer, MedR 1987, $271 \mathrm{ff}$. (273)

${ }^{36}$ BGH. NJW 1989. 2334 ff.: BGH. NJW 1980, 1905 ff,; BGH, NJW 1985, 2192: BGH, MedR 1983, 111 ff.; BGH, NJW 1972, 335 f.; Laufs, NJW 1993. $1497 \mathrm{ff}$.
} 
Berhalb der Wahrscheinlichkeit liegende Risiken für den Entschluß des Patienten zur Durchführung der Behandlung haben können. Demnach muß selbst bei extrem seitenen Zwischenfallrisiken (von $1 \%$ oder $0,5 \%$ ) die Entscheidungsfreiheit des Patienten grundsätzlich gewahrt bleiben, d. h. er muß aufgeklärt werden. Unterhalb einer solchen Komplikationsdichte von $1 \%$ kann von einer Aufklärung regelmäßig nur abgesehen werden, wenn die mögliche Folge bei einem verständigen $\mathrm{Pa}$ tienten für den Entschluß zur Erteilung der Einwilligung nicht ernsthaft ins Gewicht fallen kann. ${ }^{37}$ Es kommt nicht auf das reine Zahlenverhältnis an, sondern auf die Schwere der möglichen Komplikationen. ${ }^{38}$ Aufklärungspflichtig sind daher auch Risiken, die nur sehr selten aufzutreten pflegen, aber erheblich die körperliche Befindlichkeit des Betroffenen belasten können, insbesondere, wenn das Risiko gerade eine typische Folge der Behandlung darstellt. ${ }^{39}$

Für den Fall der Fremdbluttransfusion gilt nach einer Entscheidung des Bundesgerichtshofes ${ }^{40}$ folgendes:

„Eine HIV-Kontaminierung von Blutkonserven ist sowohl für den Empfänger des Bluts als auch für dessen Kontaktpersonen mit verheerenden Konsequenzen verbunden (...). Auch eine Transfusionshepatitis kann für den Betroffenen zu einer erheblichen gesundheitlichen Beeinträchtigung führen. Solange diese Risiken nicht ausgeschlossen werden können, ist deshalb vor einer Operation eine Aufklärung des Patienten über das Risiko einer Infektion mit Hepatitis oder AIDS durch Transfusion von Fremdblut jedenfalls immer dann erforderlich, und zwar rechtzeitig vor der Operation, wenn es für den Arzt ernsthaft in Betracht kommt, daß bei diesem Patienten intra- oder postoperativ eine Bluttransfusion erforderlich werden kann."

\section{Der Versuch einer Systematisierung der Aufklärungspflicht von Schlosshauer-Selbach}

Die Durchführung der Aufklärung bereitet dem Arzt in verschiedener Hinsicht im Einzelfall Probleme. Die Judikatur macht die Notwendigkeit der Aufklärung vom Einzelfall abhängig und stellt immer detaillierter werdende Anforderungen an den Arzt. ${ }^{41}$ Das führt, wie auch die eingangs angesprochene Diskussion während der Jahrestagung zeigt, ohne Unterlaß zu einer ständigen und erheblichen Verunsicherung der Ärzte, denn sie wissen nie genau und sie können es oft auch gar nicht wissen, ob sie bei der Aufklärung alles richtig gemacht haben oder nicht.

In der juristischen Literatur werden gelegentlich Schemata zusammengestellt, die versuchen, die maßgeblichen Grundsätze der Rechtsprechung gleichsam als Leitlinie darzustellen. Schlosshauer-Selbach ${ }^{42}$ faßt diese Grundsätze wie folgt zusammen:

\footnotetext{
${ }^{37}$ BGH, NJW 1989, 2334 ff. (2334).

${ }^{38}$ BGH, MedR 1983, 111 ff. (111).

${ }^{39}$ BGH, NJW 1980, 1905 ff. (1907); BGH, MedR 1983, $111 \mathrm{ff},(111)$; BGH,NJW 1985, $2192 \mathrm{ff} .(2192)$.

${ }^{40} \mathrm{BGH}$, NJW 1992, 743 ff. (744).

${ }^{41}$ Bodenburg, NJW 1981, 601 ff. (601).

42 Schlosshauer-Selbach, DRiZ 1982, $361 \mathrm{ff}$.
}

1. Der Arzt muß nie über beherrschbare Gefahren aufklären, also über vermeidbare Fehler (z. B. Nervenstrangverwechslung) sowie über Gefahren, die mit jedem Eingriff verbunden sind (z. B. Thrombose), weil die Kenntnis hierüber beim Patienten vorausgesetzt werden kann.

2. Er muß aber immer über mit Sicherheit eintretende typische Folgen aufklären (z.B. Verlust der Gebärfähigkeit bei Uterusextirpation).

Für den Arzt stellt sich das Problem, welchen Anforderungen er innerhalb dieser Grenze bei der Aufklärungspflicht nachkommen muß. Schlosshauer-Selbach ${ }^{43}$ stellt hierzu folgende Kriterien zusammen:

a) Wahrscheinlichkeit der Schädigung (Komplikationsdichte): Je wahrscheinlicher die konkrete Gefahr einer Schädigung ist, um so schärferen Anforderungen muß die Aufklärungspflicht genügen, um so umfassender muß der Arzt also aufklären.

b) Dringlichkeit der Behandlung:

Je weniger dringlich die Behandlung ist, um so stärker sind die Anforderungen der Aufklärungspflicht. SchlosshauerSelbach stellt eine Skala von geringer zu höchster Dringlichkeit, von der kosmetischen Behandlung, der diagnostischen Behandlung, der therapeutisch indizierten Behandlung mit mäßiger Gesundheitsbeeinträchtigung, der therapeutisch indizierten Behandlung mit erheblicher Gesundheitsbeeinträchtigung und der therapeutischen Behandlung mit vitaler lebensbedrohener Indikation auf.

c) Umfang der möglichen Schädigungen:

Je schwerwiegender im konkreten Fall mögliche Schädigungen sind, um so höhere Anforderungen werden an die Aufklärungspflicht gestellt. Über die Gefahr dauernder und erheblicher Schädigungen muß immer aufgeklärt werden.

d) Verhalten des Patienten:

Vom Patienten ungefragt muß der Arzt stets eine Aufklärung in den Grundzügen (sog. Basisaufklärung) leisten, Fragen des Patienten hat der Arzt auch bei abwegigster Fragestellung erschöpfend zu beantworten.

Schlosshauer-Selbach nimmt die Kritik an diesem System: फ़ gleich selbst vorweg und stellt fest, man könne es nicht gerade $\stackrel{\bar{\alpha}}{\alpha}$ als einen erlösenden Ariadnefaden bezeichnen. Es herrsche ein $\xi$ Case-Law, das mehr durch konkret-individuelle Einzelfallgerechtigkeit gekennzeichnet sei als durch abstrakt-generelle Rechtssicherheit. ${ }^{44}$

Für die Frage nach der Aufklärung bei der Eigenbluttransfusion gilt hiernach, daß der Arzt den Patienten darüber und über die 흥 Einzelheiten dieser Behandlung aufklären muß. Die Gefahr der Schädigung des Patienten wegen der in seinem ihm infundierten Blut enthaltenen Krebszellen ist medizinisch umstritten, $\bar{\square}$ so daß deswegen die Aufklärung unerläßlich ist.

\footnotetext{
${ }^{43}$ Schlosshauer-Selbach, a. a.O.

${ }^{44}$ Schlosshauer-Selbach. a.a.O. - vgl. dort auch zu Sonderfällen entbehrlicher Aufklärung.
} 


\section{Ausnahmen von der Aufklärungspflicht}

An dem Ergebnis der bestehenden Aufklärungspflicht ändert sich nichts, wenn man sich die Ausnahmefälle, bei denen eine Aufklärung entfallen kann, ansieht; eine Aufklärung ist nicht geboten,

- wenn der Patient bewußtlos oder aus sonstigem Grund ohne hinreichende Einsichtsfähigkeit ist und nur eine sofortige Behandlung ihn aus einem lebensbedrohenden (oder jedenfalls mit schweren Schäden verbundenen $\mathrm{Zu}$ stand) retten kann..$^{45}$

- unter engen Voraussetzungen bei der Operationserweiterung. Das gilt i.d.R. aber nur, wenn sie unvorhersehbar war und eine vitale Indikation gegeben ist, wobei diesem, wie auch dem erstgenannten Fall, die Rechtsfigur der mutmaßlichen Einwilligung zugrunde liegt. ${ }^{46}$ Es darf nur dann auf den mutmaßlichen Willen des Betroffenen abgestellt werden, wenn der Patient seinen tatsächlichen Willen nicht äußern kann.

wenn der Patient bereits ausreichend informiert ist (was genau überprüft werden muß) oder sich vertrauensvoll dem Arzt ausliefert und ausdrücklich und eindeutig auf die Aufklärung verzichtet. ${ }^{47}$

wenn die Aufklärung kontraindiziert ist, ${ }^{48}$ wenn sie also den Betroffenen seelisch schwer niederschmettern würde. Das ist allerdings umstritten und wird nur dann vom $\mathrm{BGH}$ anerkannt, wenn durch die seelische Erschütterung Leben und Gesundheit des Patienten ernstlich gefährdet sein würden.

Bei der einfachen intraoperativen Bluttransfusion (ohne Tumorzellen des Patienten) als ein Nebeneingriff unter einer Reihe anderer (potentieller) Nebeneingriffe, ${ }^{49}$ stellt sich zwar die Frage, ob der Patient, wenn er in die Operation einwilligt, sich dieses möglichen Nebeneingriffs bereits bewußt sein könnte, denn man könnte annehmen, es sei allgemein bekannt, daß der bei einem operativen Eingriff eingetretene Blutverlust durch Bluttransfusionen ausgeglichen werden muß. ${ }^{50}$ Es ist aber keineswegs sicher, ob der Patient damit auch wirklich rechnet, daß während der Operation ein Blutverlust eintritt, der eine Blutübertragung erforderlich machen kann. ${ }^{51}$ Der $\mathrm{BGH}^{52}$ hat entschieden, daß der Arzt sich nicht darauf verlassen dürfe, daß der Patient mit einer Bluttransfusion rechne oder keine nähere Aufklärung darüber wünsche und stillschweigend einwillige. Das gilt gerade bei Eingriffen, bei denen es eher unwahrscheinlich ist, daß eine Blutübertragung erforderlich wird. Hier geht der Patient noch weniger von einer solchen Nebenfolge aus, auch wenn sie nicht mit Sicherheit auszuschließen ist. ${ }^{53}$ Also muß grundsätzlich über die Möglichkeit der Bluttransfusion aufgeklärt werden, sie ist regelmäßig nicht derart unwahrscheinlich, daß die Aufklärungspflicht entfallen würde.

\footnotetext{
45 Schlosshauer-Selbach, DRiz 1982, $361 \mathrm{ff}$. (363).

46 Schlosshauer-Selbach, DRiz 1982, $361 \mathrm{ff}$. (363).

47 U/senheimer, Arztstrafrecht in der Praxis 1988, 84, Rdnr. 125, 126.

48 Ulsenheimer, Arztstrafrecht in der Praxis, 1988, 85, Rdnr. 127.

49 Laufs, NJW 1993, $149 \mathrm{ff}$. (1502).

50 BGH, NJW 1992, $743 \mathrm{ft} .(744)$.

52 BGH, NJW 1992, 743 ff. (744).

52 BGH. NIW 1992, 743 ff. (744); kritisch Weißauer, Opderbecke, MedR 1992. $308 \mathrm{ff}$.

Weißauer, MedR 1987, 272 ff. (273).
}

\section{Aufklärung in der Praxis}

Aus der Fülle von Fragen der Ärzte zur Aufklärung des Patienten sollen nachfolgend nur einige immer wiederkehrende Probleme kurz dargestellt werden, nämlich die Art und Weise der Aufklärung des Patienten, der „richtige“ Zeitpunkt der Aufklärung, die Aufklärungsschritte und die Ersatz- und Zusatzaufklärung.

\section{Die Art und Weise der Durchführung der Aufklärung}

Zu den Anforderungen an die Art und Weise der Aufklärung ist folgendes festzustellen: Der Patient muß so konkret über Wesen, Bedeutung und Tragweite des beabsichtigten Eingriffs unterrichtet werden, daß er eine eigenverantwortliche Entscheidung treffen kann, wobei es nach ständiger Rechtsprechung des $\mathrm{BGH}^{54}$ nicht als erforderlich angesehen wird, dem Patienten medizinische Einzelheiten über einen vorgesehenen ärztliche Eingriff zu eröffnen. Es wird aber verlangt, daß er „im großen und ganzen" weiß, worin er einwilligt, d. h., daß er jedenfalls wissen muß, worum es bei dem Eingriff geht und wie er die Erfolgsaussichten und Gefahren einzuschätzen hat. Der Arzt darf sich nicht mit belanglosen Redewendungen begnügen, sondern dem Patienten muß so ausreichendes Wissen vermittelt werden, daß er in die Lage versetzt wird, selbständig zu entscheiden, ob er sich dem angeratenen medizinischen Eingriff aussetzen will oder nicht..$^{55}$

Die eingehende Vermittlung der medizinisch bedeutsamen Fakten muß dabei in einer für den Laien verständlichen Form erfolgen, d. h. der Arzt soll dem Patienten keine wissenschaftliche Vorlesung halten, sondern die Aufklärung anpassen an dessen Bildungsstand, dessen gesundheitliche Verfassung, sein Alter usw. Die medizinischen Informationen sollen in diesem Informationsgespräch verständlich (.Übersetzung in die Laiensphäre") erteilt werden. ${ }^{56}$

Die Aufklärung als Teil der Heilbehandlung darf nur durch einen Arzt stattfinden und nicht durch medizinisches Hilfspersonal, also nicht durch Krankenschwestern oder medizinischtechnische Assistenten. ${ }^{57}$

\section{Der Zeitpunkt der Aufklärung}

Die Aufklärung muß nach Möglichkeit so rechtzeitig erfolgen. daß der Patient in Ruhe abwägen kann, ob er sich der Behandlung unterziehen soll: Die Aufklärung darf nicht erst auf dem Operationstisch, aber in der Regel auch nicht erst am Abend vor der Operation erfolgen, da in solchem Fall dem Patienten eine Verweigerung der Einwilligung aufgrund freier Entscheidung oft nicht mehr möglich wäre. ${ }^{58}$ Feste Richtlinien gibt es auch hier nicht, die Frage der Rechtzeitigkeit richtet sich u.a.

\footnotetext{
54 BGH, MedR 1990, $264 \mathrm{ff},(264)$

55 BGH, MedR 1990, $264 \mathrm{ff}$. (264).

56 Bodenburg, NIW 1981, $601 \mathrm{ff}$. (602): BGH, MedR 1990, $264 \mathrm{ff}$. (266).

57 Schlosshauer-Selbach, DRiz 1982, 361 ff. (364).

58 Scholz, MDR 1996, 649ff. (650),
} 
nach der Dringlichkeit des Eingriffs, wobei bei nicht vitaler Indikation, jedenfalls bei schweren Eingriffen, allgemein von einem Zeitabstand von drei Tagen ausgegangen wird. ${ }^{59}$

Die Gerichte haben sich mit dem Aufklärungszeitpunkt immer wieder zu befassen; z. B. stellte der BGH dazu kürzlich fest:

„Es bedarf keiner näheren Darlegung, daß die Einwilligung der Klägerin unwirksam ist, wenn es hierfür auf den Zeitpunkt der Unterzeichnung der Einwilligungserklärung ankommt. Zutreffend macht die Revision geltend, daß eine Einwilligungserklärung unter derartigen Umständen, nämlich auf dem Weg zum Operationssaal und unter dem Einfluß einer Beruhigungsspritze, nicht für wirksam erachtet werden könne, zumal die Klägerin durch das auf Unterzeichnung der Einwilligungserklärung gerichtete Drängen des Arztes, man könne andernfalls die Operation auch unterlassen, massiv eingeschüchtert und in ihrer Entscheidungsfreiheit beeinträchtigt worden sei. Bei einem solchen Vorgehen wird das Selbstbestimmungsrecht des Patienten als Grundlage einer wirksamen Einwilligung nicht ausreichend gewahrt." 60

\section{Das Problem der Aufklärungsblätter}

Dem Unbehagen und der Unsicherheit vieler Ärzte, die „richtig“ aufklären wollen und die nur zu oft erst hinterher im Prozeß gesagt bekommen, wie sie es nach Meinung des Gerichts besser hätten machen sollen, bietet das Unwesen der überall verwendeten Aufklärungsblätter nur scheinbar einen Ausweg: gegen die Flut von Merkblättern und Aufklärungsformularen ${ }^{61}$ sind erhebliche Bedenken ${ }^{62}$ anzumelden: Im Wege der Rationalisierung der ärztlichen Arbeit wird das Interesse des Patienten an einfühlsamer und individueller Aufklärung zurückgedrängt. Der Patient wird mit perfektionistisch ausgefeilten Texten konfrontiert, die er in seiner Aufregung und als medizinischer Laie kaum noch oder gar nicht zu begreifen vermag. Das beschädigt die Basis des Vertrauensverhältnisses zwischen Arzt und Patient oder läßt es oft gar nicht erst entstehen; der Patient gerät, wenn er schon bei der Aufnahme sich gezwungen sieht, Honorarvereinbarungen zu unterschreiben und zahlreiche andere Zettel, bevor er den Arzt überhaupt trifft, und wenn ihm später jemand weitere Aufklärungsformulare auf das Krankenbett wirft, in eine Konfliktsituation zum Arzt, die Prozesse geradezu provoziert. Dann kommt noch ein falsches Wort eines Arztes oder sonst ein Mißverständnis dazu, schon beauftragt der Patient seinen Anwalt und es entstehen jahrelang andauernde Straf- und Zivilprozesse.

Der urologische Ausgangsfall oben zeigt ein weiteres Problem auf: Es besteht generell die Gefahr der Verwendung alter Formulare, die vielleicht schon seit langem irgendwo in der Klinik liegen oder der Formularfabrikant ist nicht auf dem aktuellen Stand. Bei Verwendung eines Formulars muß der Arzt prüfen,

\footnotetext{
${ }^{59}$ BGH, MedR 1983, 73 ff. (76).

${ }^{60} \mathrm{BGH}, \mathrm{NJW} 1998,5.1784 \mathrm{f}$.

${ }^{61}$ dazu Laufs, NJW 1990, $1505 \mathrm{ff}$.

62 Deutsch, NJW 1982, 2585 ff. (2588); Scholz, MDR 1996, 649 ff . (653).
}

ob es in allen Details auf den Einzelfall paßt. Diese Formularkontrolle entfällt bei dem ohnehin obligatorischen Aufklärungsgespräch, die Kontrolle bedeutet nur Zusatzarbeit. Der Arzt ist für die Vollständigkeit und Richtigkeit der verwendeten Formulare verantwortlich. Die Einzelfallproblematik kann in einem Formular nicht ausreichend berücksichtigt werden. Eine ausreichende und umfassende Information des Patienten ist aber erforderlich.

Viele Richter stehen den Formularen sehr skeptisch gegenüber. Die Formulare haben keineswegs die Entlastungsfunktion, die die Formularhersteller gegenüber den Ärzten oft behaupten. Zwar wird hier nicht bestritten, daß sie als schriftliche Zusatzaufklärung eine wertvolle Unterstützung der mündlichen Aufklärung sein können. Formularblätter enthalten jedoch ein großes rechtliches Risiko und vermitteln eine trügerische Sicherheit, entscheidend ist die vertrauensvolle Unterredung zwischen Arzt und Patient, ${ }^{63}$ denn beweisrechtlich helfen Einwilligungserklärungen nur, wenn sich der vollständige Ablauf der mündlichen Aufklärung daraus ergibt. ${ }^{64}$ Die Aufklärung muß daher in einem Gespräch stattfinden, bei dem der Arzt zuerst in Erfahrung bringen soll, wie weit der Patient informiert zu werden wünscht, um dann die Aufklärung daran auszurichten. ${ }^{65}$ Die Aufklärung läßt sich durch ihre Dokumentation oder auch in einigen Fällen durch die Hinzuziehung eines Mitarbeiters als etwaigen Zeugen ausreichend beweiskräftig machen. ${ }^{66}$ Problematisch hingegen erscheint die vom Patienten evtl. sogar mit Druck abverlangte Quittierung der erfolgten Aufklärung, denn daraus ergibt sich nichts über den Inhalt, also nichts dazu, worüber der Patient konkret informiert worden sein soll, vor allem nichts darüber, ob er das alles verstanden hat oder nicht.

Zur Beweissituation der Ärzte hat z.B. das OLG Karlsruhe in Einklang mit der sonstigen neueren Rechtsprechung festgestellt:

„Auch der Senat erachtet es aufgrund der vom Kläger unterschriebenen Einverständniserklärung i.V. mit der Aussage des Zeugen Dr. S. für nachgewiesen, daß der Kläger vor der Operation aufgeklärt worden ist. Zwar hat der Zeuge keine konkrete Erinnerung an das Aufklärungsgespräch gehabt. Er hat jedoch in nachvollziehbarer und in sich stimmiger Weise die übliche Vorgehensweise bei einem Aufklärungsgespräch vor einer derartigen Operation geschildert und zugleich bekräftigt, daß er sich ganz sicher sei, daß dieses Programm immer eingehalten worden sei. Dies genügt zur Überzeugungsbildung, denn an den dem Arzt obliegenden Beweis der ordnungsgemäßen Aufklärung des Patienten dürfen keine unbilligen oder übertriebenen Anforderungen gestellt werden." 6 ?

\footnotetext{
${ }^{63}$ Laufs, NJW 1990, 1505 ff. (1509).

${ }^{64} \mathrm{BGH}$, MedR 1996, $213 \mathrm{ff}$. (213).

${ }^{65}$ Deutsch. NJW 1982, 2585 ff. (2588).

to Schlosshauer-Selbach, DRiZ 1982, $361 \mathrm{ff}$. (365).

67 OLG Karlsruhe NJW 1998, S. 1800 mit Hinweis auf: BGH. NJW 1984, $1403=$ VersR, 386. (387); NJW 1985, $1399=$ VersR 1985, 361 (362); OLG Oldenburg. VersR 1995, 1194 (1195): vgl. auch BGH, NJW 1994. $3009(3010)=$ VersR 1994,1235
} 


\section{Ersatz- und Zusatzaufklärung}

Bei Minderjährigen und volljährigen aber willensunfähigen Patienten muß eine Ersatzaufklärung erfolgen, wenn der Patient die Aufklärung zu verstehen nicht in der Lage ist. Auch hier können sich schwierige Probleme ergeben, die nur im Einzelfall und nicht pauschal gelöst werden können. Auszugehen ist dabei von folgendem Ansatzpunkt: Die Einwilligung ist keine rechtsgeschäftliche Willenserklärung, so daß es auf die bürgerlich-rechtliche Geschäftsfähigkeit nicht ankommt, sondern entscheidend ist vielmehr, daß der Patient die entsprechende natürliche Einsicht-, Urteils- und Verständnisfähigkeit besitzt, um im konkreten Fall die ärztliche Maßnahme, ihre Folgen und das insoweit bestehende Risiko zu ermessen. Das muß der Arzt in jedem Einzelfall prüfen. ${ }^{68}$ Ab welchem Alter der Minderjährige die nötige Einsichtsfähigkeit hat, läßt sich nicht generell durch bestimmte Altersgrenzen, sondern nur anhand der Umstände des konkreten Falles festlegen, wobei immerhin weitgehend Einigkeit darüber besteht, daß Minderjährige unter 14 nicht, Minderjährige kurz vor Vollendung des 18 . Lebensjahres dagegen regelmäßig schon einwilligungsfähig und damit selbst aufklärbar sind. ${ }^{69}$

Fehlt dem Patienten die volle eigene Einsichtsfähigkeit, dann muß die Einwilligung des gesetzlichen Vertreters eingeholt und dieser muß aufgeklärt werden. Bei Minderjährigen sind das i.d.R. die Eltern ( $\$ 1629$ BGB), bei nicht voll einwilligungsfähigen Volljährigen ist es der zuständige Betreuer $(\S \S 1898 \mathrm{ff}$. BGB). ${ }^{70}$ In schwierigen Fällen, z. B. wenn aufgrund der Behandlung die Gefahr des Todes oder eines schweren oder langdauernden Schadens für den Betreuten besteht, muß darüber hinaus zusätzlich eine Genehmigung des Vormundschaftsgerichtes eingeholt werden. ${ }^{71}$

\section{Die Zuständigkeit für die Durchführung der Aufklärung bei der intraoperativen Bluttransfusion}

Für die intraoperative Blutübertragung ist nach ständiger Übung und allgemeiner Auffassung der Anästhesist zuständig. ${ }^{72}$ Da aufklärungspflichtig grundsätzlich der den Eingriff eigenverantwortlich durchführende Arzt ist, ${ }^{73}$ ist bei der Bluttransfusion der Anästhesist auch für die Aufklärung zuständig. Die Aufklärung über die Blutübertragung ist, weil sie zugleich, wie oben ausgeführt, einen operativen Nebeneingriff darstellt, Teil der Risikoaufklärung zur Operation, deren Durchführung dem Operateur obliegt. Deshalb liegt die Aufklärungspflicht zur Bluttransfusion sowohl beim Operateur als auch beim Narkosearzt.

Beide Ärzte müssen den Patienten nicht persönlich aufklären. ${ }^{74} \mathrm{Im}$ Wege der üblicherweise bestehenden Arbeitsteilung in Kliniken kann die Durchführung der Aufklärung delegiert und einem der beiden Ärte oder auch einem anderen Arzt, et- wa dem Stationsarzt, übertragen werden, womit in diesem Fall der beauftragte Arzt zuständig ist. ${ }^{75}$ Voraussetzung für die Aufklärungsdelegation ist stets eine klare Absprache und Kompetenzverteilung, damit die Pflicht zur Information des Patienten auch erfüllt wird und nicht in den Verästelungen vertikaler und horizontaler ärztlicher Arbeitsteilung schließlich untergeht. ${ }^{76}$ Zudem darf sich weder der Operateur noch der Anästhesist darauf verlassen, daß der Patient unterrichtet worden ist, sondern muß sich stets darüber vergewissern. ${ }^{77}$ Die Aufgabendelegation durch Arbeitsteilung entbindet auch hier nicht von der Verantwortung zu prüfen, ob eine ordnungsgemäße Information des Patienten tatsächlich stattfindet oder stattgefunden hat.

\section{Ergebnis}

Ärzte können nur versuchen, sich vor Aufklärungsfehlern zu schützen; ob sie alles richtig gemacht haben, erfahren sie u.U. im nachhinein dann von den Gerichten. Als Richtlinie zur Vermeidung eines Konflikts kann man zweierlei raten: Jeder Patient soll soweit als möglich über alles aufgeklärt werden, was mit seiner Behandlung zusammenhängt. Aus anwaltlicher Erfahrung im Zusammenhang mit der anwaltlichen Aufklärung von Mandanten läßt sich feststellen, daß eine sachliche Aufklärung, die u.U. auch öfter wiederholt wird, grundsätzlich und regelmäßig zu einem tragfähigen Vertrauensverhältnis führt und Prozesse verhindert. Die Situation, in der sich der Patient befindet, wenn er in die Klinik kommt, bedarf in vielen Fällen einer erheblichen Verbesserung, denn sie löst oft den Arzt-Patientenkonflikt erst richtig aus, wie sich aus vielen Gerichtsakten zu Kunstfehlerfällen ergibt. Die Aufklärungsproblematik ist nur ein Teilbereich des Arzt-Patientenkonflikts; erst die Betrachtung der gesamten Beziehung zwischen Arzt und Patient führt in vielen Fällen zur Kenntnis der eigentlichen Ursache des Streits, die oft in den folgenden Umständen liegt: In Kliniken fühlen sich Patienten nicht selten durch die dortige Situation geradezu provoziert: Stundenlanges Warten, das energische Verlangen, zahlreiche Zettel zu unterschreiben, durch Nachtdienste gestreßte Ärzte usw. müssen in Verbindung mit der Erkrankung zum Zorn der Patienten führen. Wenn Ärzte dann noch Patienten mit lockeren Sprüchen aufregen (z.B.: „Wer in meinem Wartezimmer ist, ist krank - wer krank ist, hat Zeit“), trägt nicht zu einer die Aufklärungsproblematik entschärfenden Situation bei, im Gegenteil.

\section{Dr. Klaus Wasserburg}

Adam-Karrillon-Str. 23 55118 Mainz

\footnotetext{
${ }^{68}$ Kollhoser, Kubillus, JA 1996, $339 \mathrm{ff}$. (340).

69 Ulsenheimer. Arztstrafrecht in der Praxis, 1988, S. 76. Rdnr. 109.

${ }^{70}$ Kollhoser, Kubillus, JA 1996, 339 ff. (340).

${ }^{71}$ Kollhoser, Kubillus, JA 1996, $339 \mathrm{ff}$. (340): Scholz. MDR 1996, $649 \mathrm{ff}$. (651).

72 Weißauer, MedR 1987, 272 ff. (274).

73 Eser, in: Schönke/Schröder, \$223 Rdnr. 40.

74 Wasserburg, Akt. Urol. 1990, S. 201.
}

\footnotetext{
75 Ulsenheimer. Arztstrafrecht in der Praxis, 1988, S. 73 ff., Rdnr. 104 ff.

76 Ulsenheimer, Artzstrafrecht in der Praxis, 1988, S. 75, Rdnr. 105.

77 OLG Hamburg. JR 1975, 511 ff. mit Anmerkung Rudolphi. 513.
} 